\title{
Wer sind die Betroffenen der Krise? Parallelen und Unterschiede zur vorangegangenen Krise
}

Infolge der weltweiten Finanzmarktkrise erlebt Deutschland ab dem 1. Quartal 2008 die größte wirtschaftliche Rezession seit dem Zweiten Weltkrieg. Gleichzeitig sind die Folgen auf dem Arbeitsmarkt bisher vergleichsweise maßvoll. Während die Entwicklung der Wirtschaftsleistung in dieser aktuellen Krise deutlich schlechter war als in der letzten Konjunkturkrise, die im 1. Quartal 2001 begann und seit Herbst 2001 auf dem Arbeitsmarkt sichtbar wurde, ist die Entwicklung auf dem Arbeitsmarkt heute günstiger. Aber gilt dies auch für unterschiedliche Arbeitsmarktbereiche, Beschäftigungsformen und Personengruppen am Arbeitsmarkt? ${ }^{1}$

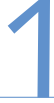 \\ Einleitung}

Die weltweite Finanzmarktkrise brachte Deutschland die größte wirtschaftliche Rezession der Nachkriegszeit. Das Bruttoinlandsprodukt verzeichnete seinen stärksten Rückgang, allerdings bisher ohne entsprechend schwere Folgen auf dem Arbeitsmarkt.

Während die Entwicklung der Wirtschaftsleistung in dieser aktuellen Krise deutlich schlechter war als in der letzten Konjunkturkrise, die auf das Platzen der sogenannten „Dotcom-Blase“ im 1. Quartal 2001 folgte, ähneln sich die Entwicklungen der Erwerbstätigkeit im Allgemeinen und der sozialversicherungspflichtigen Beschäftigung im Besonderen in beiden Krisenverläufen. Bei der registrierten Arbeitslosigkeit ist tendenziell sogar eine bessere Entwicklung in der aktuellen Krise zu beobachten (Abbildung 1). So stieg sie im Jahr 2002 gegenüber dem Vorjahr um 5,4 \%, im Vergleich der Jahre 2008 und 2009 lag die Steigerungsrate hingegen nur bei 4,8\% (vgl. dazu Tabelle 2 im Abschnitt 3.1).

Möller (2010) sowie Möller/Walwei (2009) gehen der Frage nach, warum der deutsche Arbeitsmarkt bisher vergleichsweise wenig von der Krise betroffen ist. Eine Erklärung für dieses Phänomen ist, dass - im Gegensatz zur vorangegangenen Krise und von der Politik begünstigt - die Kurzarbeit durch die Betriebe aktuell in weitaus stärkerem Maße in Anspruch genommen wird. Eine andere Erklärung liegt in institutionellen Regelungen wie Arbeitszeitkonten, die darauf ausgerichtet sind, innerhalb der Firmen einen großen Spielraum für die Absorption ökonomischer

\section{Abb. 1: Entwicklung der saisonbereinigten Arbeitslosigkeit im Krisenvergleich}

- in \%, 1. Quartal 2001 und 1. Quartal $2008=100-$

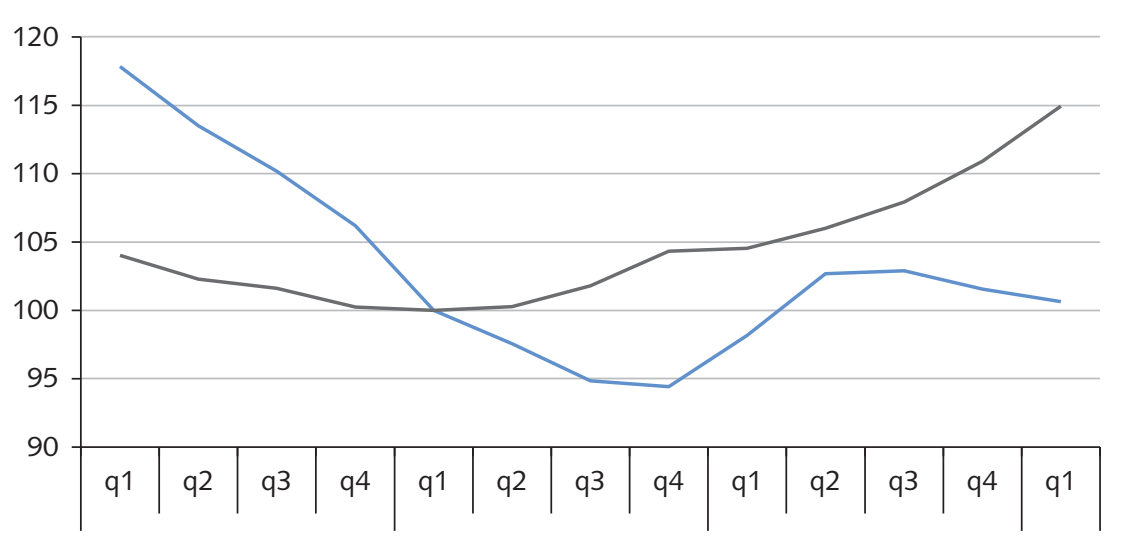

— aktuelle Krise (q1-2007 bis q1-2010)

vorangegangene Krise (q1-2000 bis q1-2003)

Quelle: Bundesagentur für Arbeit, Berechnungen der Autoren.

WSI MITTEILUNGEN

Schocks zu schaffen. Zudem wird argumentiert, dass auch die Arbeitsmarktreformen und das Verhalten der Sozialpartner die Anpassungsmöglichkeiten bei einem temporären Nachfrageausfall begünstigen (Möller 2010). ${ }^{2}$

Auffallend ist, dass die Ausgangslage am Arbeitsmarkt aktuell besser war als bei der vorangegangenen Krise. Zwar lag die Gesamtzahl der sozialversicherungspflichtig Beschäftigten im Jahr 2008 mit knapp 28 Mio. etwas unter der Zahl von

1 Wir danken Alexander Herzog-Stein, Ulrike Stein sowie den Gutachtern im Referee-Prozess für wertvolle Hinweise und Anregungen

2 Der besonderen Rolle der Kurzarbeit bei der Bewältigung der Krise widmen sich auch die Beiträge von Crimmann/Wießner (2009); BA (2009a) sowie Bogedan in diesem Heft.
Martin Rosemann, Dr., ist wissenschaftlicher Referent und Projektleiter im Bereich Arbeitsmärkte und soziale Sicherung am Institut für Angewandte Wirtschaftsforschung (IAW) in Tübingen und Leiter des Kompetenzzentrums Mikrodatenbasierte Politikberatung. Arbeitsschwerpunkt: Evaluation von arbeitsmarkt- und sozialpolitischen Maßnahmen und Instrumenten. e-mail: martin.rosemann@iaw.edu Andrea Kirchmann ist wissenschaftliche Referentin und Projektleiterin im Bereich Arbeitsmärkte und soziale Sicherung am IAW in Tübingen. Arbeitsschwerpunkt: Evaluation von arbeitsmarkt- und sozialpolitischen Maßnahmen und Instrumenten. e-mail: andrea.kirchmann@iaw.edu Unter Mitarbeit von Stefanie Baller und Sarah Eichmeyer. 
2001 mit 28,2 Mio. Die Gesamtzahl der Erwerbstätigen lag jedoch im Jahr 2008 mit 40,7 Mio. um über eine Million über dem Stand von 2001. Die Zahl der registrierten Arbeitslosen lag im September 2008 mit etwas über 3 Mio. deutlich unter dem Wert von 3,7 Mio. im September 2001. ${ }^{3}$ Zudem war sowohl für die registrierte Arbeitslosigkeit (Abbildung 1) als auch für die Erwerbstätigkeit und die sozialversicherungspflichtige Beschäftigung die Entwicklung vor Beginn der aktuellen Krise besser als vor der letzten Krise. ${ }^{4}$

Es existiert bereits Literatur zu den Auswirkungen der aktuellen Krise auf verschiedene Arbeitsmarktbereiche und Personengruppen (z. B. BA 2009b; Bach et al. 2009; IW 2010). Heckmann et al. (2009) untersuchen auf Basis einer repräsentativen Befragung von Unternehmen, welche Bereiche der deutschen Wirtschaft im 2. Quartal 2009 von der Wirtschaftskrise betroffen waren und mit welchen Maßnahmen versucht wurde, der Krise zu begegnen. Schwengler/Loibl (2010) stellen dar, wie sich der vergangene Aufschwung und die aktuelle Krise jeweils in unterschiedlichen Regionen auswirken. Bogedan et al. (2009) beschäftigen sich auf Basis der WSI-Betriebsrätebefragung 2009 mit betrieblicher Beschäftigungssicherung in der Krise.

Allerdings fehlen im Hinblick auf die Betroffenheit unterschiedlicher Personengruppen und Arbeitsmarktbereiche bisher umfassende Vergleiche zur vorangegangenen Krise. Der vorliegende Artikel will diese Lücke schließen. Ausgehend davon, dass mit Blick auf die aggregierten Arbeitsmarktzahlen die Entwicklungen in der aktuellen Krise günstiger ausfielen als in der vorangegangenen, konzentriert sich unser Beitrag auf die Frage, ob dies auch für unterschiedliche Arbeitsmarktbereiche, Beschäftigungsformen und Personengruppen am Arbeitsmarkt gilt.

Unsere zentrale These ist, dass nur eine solch differenzierte Betrachtung zeigt, was die Krise letztlich für den deutschen Arbeitsmarkt bedeutet. Ausgangspunkt der Krise war der Finanzmarktsektor - vorrangig in den USA. Deshalb wäre es naheliegend, dass auch in Deutschland dieser Wirtschaftsbereich in besonderer Weise betroffen ist. Ebenso naheliegend ist es jedoch, dass sich Probleme auf den Finanzmärkten über die Finanzierungsstrukturen von Unternehmen relativ schnell auf andere Wirtschaftsbereiche übertragen, wobei investitionsintensive Branchen besonders betroffen sein dürften. Gleichzeitig sind die Übertragungsmechanismen von Konjunkturkrisen zu beachten (SVR 2001): Neben der wachsenden Verflechtung der Finanzmärkte spielt für die Bundesrepublik als „Exportweltmeister“ dabei nach wie vor die Entwicklung der Exportnachfrage eine zentrale Rolle. Zunehmende Bedeutung bei der Krisenübertragung haben jedoch auch die deutschen Direktinvestitionen im Ausland. Beides spricht dafür, dass in besonderem Maße die Branchen mit den höchsten Auslandsumsätzen von der Krise betroffen sind, wobei Verflechtungen mit den USA eine besondere Bedeutung zukommt.

Ausgehend von diesen Überlegungen vermuten wir, dass die regionale Betroffenheit dort besonders hoch ist, wo die exportund investitionsintensiven Branchen stark vertreten sind. Traditionell sind Männer in stärkerem Maße als Frauen in den exportorientierten Branchen des Verarbeitenden Gewerbes beschäftigt, was für eine stärkere Krisenbetroffenheit der Männer im Vergleich zu den Frauen spricht. Personen mit Migrationshintergrund und vor allem gering qualifizierte Arbeitskräfte gehören zu den traditionellen Problemgruppen am Arbeitsmarkt. Es ist zu vermuten, dass sie in besonderem Maße unter der Krise $\mathrm{zu}$ leiden haben. In Krisenzeiten gehen Neueinstellungen ebenso zurück wie die Ausbildungstätigkeit, was besonders die jungen (unter 25 Jahren) Arbeitsmarktteilnehmer negativ tangiert. Von Älteren konnten sich Betriebe in der Vergangenheit gerade in konjunkturellen Krisen aufgrund von Vorruhestandsregelungen und längeren Ansprüchen auf Arbeitslosengeld leichter trennen. Zu vermuten ist, dass dies zumindest in diesem Ausmaß aktuell nicht mehr so funktionieren kann wie noch in der vergangenen Krise.

Als Erstes von Beschäftigungsverlust betroffen sind traditionell die Randbelegschaften in Form von Leiharbeit, geringfügiger und befristeter Beschäftigung. Bei der geringfügigen Beschäftigung könnte jedoch ein gegenteiliger Effekt dadurch ausgelöst werden, dass Branchen, in denen das sozialversicherungspflichtige Normalarbeitsverhältnis stark dominiert (Branchen des Verarbeitenden Gewerbes) stärker betroffen sein könnten als Branchen mit einem höheren Anteil an geringfügiger Beschäftigung (Dienstleistungsbereich). Analog dazu dürfte in den besonders stark betroffenen Branchen der Teilzeitanteil geringer sein, sodass mit einer besseren Entwicklung der Teilzeitbeschäftigung zu rechnen ist.

Unsere Schlussfolgerungen ziehen wir aus einem Vergleich der aktuellen Krisenentwicklung mit der Entwicklung der vorangegangenen Krise. Die Bestimmung des jeweiligen Ausgangszeitpunkts für die beiden Krisen wird gemäß dem Vorgehen des Sachverständigenrats anhand des Konzepts der Outputlücken (SVR 2008) vorgenommen. Daraus ergibt sich für den Beginn der vorangegangenen Krise das 1. Quartal 2001, für den Beginn der aktuellen Krise das 1. Quartal 2008, auch wenn die Krise erst ab dem 4. Quartal 2008 auf dem Arbeitsmarkt erkennbar wurde.

Davon ausgehend betrachten wir für die aktuelle Krise den Zeitraum vom 1. Quartal 2008 bis zum 1. Quartal 2010 sowie für die vorangegangene Krise vom 1. Quartal 2001 bis zum 1. Quartal 2003. ${ }^{5}$ Vergleiche zwischen unterschiedlichen Wirtschaftszweigen, Regionen, Personengruppen und Beschäftigungsformen sowie insbesondere auch zwischen der aktuellen und der vorangegangenen Krise werden ermöglicht, indem eine Normierung auf den jeweils betrachteten Ausgangszeitpunkt erfolgt. Ergänzend wird auch jeweils die Entwicklung im Jahr unmittelbar vor dem Krisenbeginn betrachtet.

In Abschnitt 2 analysieren wir zunächst die unterschiedliche sektorale und regionale Betroffenheit. Anschließend wenden wir uns in Abschnitt 3 der Frage

3 Saisonbereinigt lag die registrierte Arbeitslosigkeit im August 2001 mit 3,9 Mio. ebenfalls deutlich über dem Wert vom August 2008 mit 3,2 Mio. Ein Vergleich der beiden Jahre ist allerdings problematisch, weil sich die Arbeitsmarktstatistik im Zuge der Einführung des SGB II zum 1.1.2005 stark verändert hat. Durch die Einbeziehung der erwerbsfähigen ehemaligen Sozialhilfeempfänger in die registrierte Arbeitslosigkeit ab 2005 dürfte das Ausmaß der tatsächlichen Arbeitslosigkeit im Jahr 2001 stärker unterschätzt worden sein als im Jahr 2008.

4 Die Frage, inwiefern die insgesamt bessere Entwicklung ebenso wie die bessere Ausgangslage eine Folge der durchgeführten Arbeitsmarktreformen ist, diskutieren Kohns und Sturn/van Treeck in diesem Heft.

5 Wir nutzen Monatsdaten für die registrierte $\mathrm{Ar}$ beitslosigkeit, die Erwerbstätigkeit insgesamt sowie für Kurz- und Leiharbeit; Quartalsdaten für die saisonbereinigte registrierte Arbeitslosigkeit, die sozialversicherungspflichtige Beschäftigung und die geringfügige Beschäftigung. 
zu, welche Personengruppen aus welchen Gründen besonders stark von der Krise tangiert werden. Abschnitt 4 fokussiert auf die unterschiedliche Entwicklung verschiedener Beschäftigungsformen, bevor in Abschnitt 5 die zentralen Ergebnisse zusammengefasst werden.

\section{Sektorale und regionale Betroffenheit}

\subsection{EXPORTORIENTIERTE BRANCHEN VON DER KRISE GETROFFEN}

Von der aktuellen Wirtschaftskrise sind insbesondere die exportintensiven Bereiche des Verarbeitenden Gewerbes betroffen. Dazu gehören Maschinenbau, Fahrzeugbau sowie die chemische und pharmazeutische Industrie. Einbrüche bei der sozialversicherungspflichtigen Beschäftigung seit Beginn der Krise im Herbst 2008 sind auch im Kfz-Handel und der Nachrichtenübermittlung zu beobachten.

\subsection{WEITERHIN ZUWÄCHSE BEI DIENSTLEISTUNGEN}

In weiten Teilen des Dienstleistungssektors lassen sich auch nach Beginn der Krise weiterhin Beschäftigungszuwächse beobachten. Hierzu gehören vor allem die Bereiche Beherbergung und Gastronomie, Erziehung und Unterricht sowie das Gesundheitswesen einschließlich Heimen und das Sozialwesen. Interessant ist zudem, dass Kredit- und Versicherungsgewerbe zwar mit Beginn der Krise einen leichten Beschäftigungsabbau zu verzeichnen hatten, sich aber relativ schnell wieder erholt haben.

Auch in der vorangegangenen Krise gab es vor allem Beschäftigungsverluste in der Industrie, während sich die Dienstleistungen überwiegend weiterhin positiv entwickelten. Die Beschäftigung nahm in der chemischen Industrie, bei der Herstellung von Gummi- und Kunststoffwaren, im Maschinenbau, im Kraftfahrzeughandel und in der Nachrichtenübermittlung ab. Allerdings war im Gegensatz zur aktuellen Krise die Entwicklung im Fahrzeugbau positiv. Zudem waren seinerzeit im Unterschied zur aktuellen Entwicklung vor allem die Baubranche und die Energie- und
Wasserversorgung von Beschäftigungsverlusten betroffen. ${ }^{6}$

\subsection{BESSERE ENTWICKLUNG IM OSTEN}

Im Hinblick auf die Entwicklung der sozialversicherungspflichtigen Beschäftigung gibt es in der aktuellen Krise zwar kaum Unterschiede zwischen Ost- und Westdeutschland. Allerdings war die Entwicklung sowohl vor als auch während der letzten Krise in Ostdeutschland schlechter als in Westdeutschland. So hat die sozialversicherungspflichtige Beschäftigung 2002 im Vergleich zu 2001 in Ostdeutschland um fast $3 \%$ abgenommen, während sie in Westdeutschland nur geringfügig um 0,4 \% zurückging. Diese Entwicklung fällt in der aktuellen Krise besser aus als in der vorangegangenen - im Osten eindeutig, im Westen tendenziell (Tabellen 1 und 2).

Während die Entwicklung der saisonbereinigten Arbeitslosigkeit vor Beginn der aktuellen Krise in Westdeutschland besser war als in Ostdeutschland, ist sie seit Beginn der Krise im Westen eindeutig schlechter als im Osten. Im Osten ist die registrierte Arbeitslosigkeit im Jahr 2009 im Vergleich zu 2008 sogar um knapp $2 \%$ gesunken, während im Westen ein Anstieg von über $8 \%$ zu beobachten ist (Tabelle 2). In Ostdeutschland war sowohl zwischen dem 1. Quartal 2008 und dem 1. Quartal $2009(-5 \%)$ als auch zwischen dem 1. Quartal 2009 und dem 1. Quartal 2010 (-4\%) die registrierte Arbeitslosigkeit rückläufig, während sie in Westdeutschland in beiden Zeiträumen (+0,5\% und $+4,9 \%$ ) anstieg (Tabelle 1). Im Jahr 2009 sind zudem die Zugänge in Arbeitslosigkeit in Westdeutschland deutlich stärker angestiegen als in Ostdeutschland. ${ }^{7}$

Die stärkere Betroffenheit Westdeutschlands bei der registrierten Arbeitslosigkeit war auch in der letzten Krise zu beobachten, obwohl sie damals auch im Osten anstieg. Während in Ostdeutschland somit die Entwicklung der Arbeitslosigkeit in allen betrachteten Zeiträumen in der aktuellen Krise besser ausfiel als in der vergangenen, kann dies für Westdeutschland nicht uneingeschränkt gesagt werden: Aktuell war der Anstieg der Arbeitslosigkeit sowohl im ersten als auch im zweiten Krisenjahr niedriger als in den gleichen Zeiträumen der vorherigen Krise (Tabelle 1), jedoch war die Zunahme der durch- schnittlichen registrierten Arbeitslosigkeit im Vergleich der Jahre 2008 und 2009 mit über $8 \%$ etwas höher als im Vergleich der Jahre 2001 und 2002 mit unter 8 \% (Tabelle 2).

Hinzu kommt, dass die Kurzarbeit in der aktuellen Krise in Westdeutschland deutlich stärker zugenommen hat als in Ostdeutschland. Gleiches gilt für die Summe aus Arbeitslosen und Kurzarbeitern. Dass Kurzarbeit ein westdeutsches Phänomen ist, kann für die letzte Krise nicht in diesem Ausmaß beobachtet werden ( $\mathrm{Ta}$ bellen 1 und 2).

Westdeutschland ist somit deutlich stärker von der aktuellen Krise am Arbeitsmarkt betroffen als Ostdeutschland - im Vergleich zur vorangegangenen Krise. In diesem Ausmaß stellt das ein neues Phänomen dar. Allerdings kann dieser Effekt vor allem darauf zurückgeführt werden, dass die von der aktuellen Krise in besonderem Maße betroffenen Branchen in Ostdeutschland eine geringere Bedeutung haben als in Westdeutschland.

In den besonders von der Krise betroffenen Branchen sind sowohl in West- als auch in Ostdeutschland Beschäftigungsverluste zu beobachten. Dabei ist die Entwicklung seit Beginn der Krise in Ost und West ähnlich.

\subsection{INDUSTRIESTANDORTE IM SÜDEN VERLIEREN}

Ein Blick auf die einzelnen Bundesländer zeigt, dass vor allem diejenigen besonders tangiert sind, in denen die von der Krise besonders betroffenen Branchen am stärksten vertreten sind. So weisen Bayern und Baden-Württemberg seit Beginn der Krise die stärksten Anstiege bei der registrierten Arbeitslosigkeit auf. Eine differenziertere Betrachtung einzelner Kreise und kreisfreier Städte (Schwengler/Loibl 2010) bestätigt dieses Bild. Die Kurzarbeit variiert deutlich zwischen den Bundesländern und Regionen. So war der Süden stärker von Kurzarbeit betroffen als der Norden.

6 Ein direkter Vergleich der Entwicklungen in einzelnen Branchen zwischen den beiden Krisen ist nicht möglich, weil ab 2003 eine neue Wirtschaftszweigsystematik eingeführt wurde.

7 Weniger eindeutig ist dagegen das Bild bei den Abgängen aus der Arbeitslosigkeit, die zudem von starken saisonalen Schwankungen geprägt sind. 
Tabelle 1: Veränderungsraten zentraler Indikatoren für die aktuelle und die vorangegangene Krise im Vergleich - in \% -

\begin{tabular}{|c|c|c|c|c|c|}
\hline & & \multicolumn{2}{|c|}{$\begin{array}{c}\text { Aktuelle Krise } \\
\text { Veränderung zwischen den jeweils ersten } \\
\text { Quartalen }{ }^{8}\end{array}$} & \multicolumn{2}{|c|}{$\begin{array}{c}\text { Vorangegangene Krise } \\
\text { Veränderung zwischen den jeweils ersten } \\
\text { Quartalen }\end{array}$} \\
\hline & & 2008 zu 2009 & 2009 zu $2010^{9}$ & 2001 zu 2002 & 2002 zu 2003 \\
\hline \multirow[t]{9}{*}{ Registrierte Arbeitslose } & Insgesamt & $-1,5$ & 1,9 & 4,4 & 9,4 \\
\hline & Männer & 3,9 & 5,1 & 7,1 & 11,4 \\
\hline & Frauen & $-7,3$ & $-2,0$ & 1,1 & 6,8 \\
\hline & U25 & 2,7 & $-2,6$ & 11,7 & 10,5 \\
\hline & Ü55 & 16,2 & 10,5 & $-16,0$ & $-8,2$ \\
\hline & Deutsche & $-1,6$ & 1,9 & 3,8 & 9,4 \\
\hline & Ausländer & $-0,7$ & 2,1 & 8,9 & 9,7 \\
\hline & West & 0,5 & 4,9 & 6,5 & 12,0 \\
\hline & Ost & $-5,0$ & $-4,0$ & 1,2 & 5,3 \\
\hline \multirow[t]{5}{*}{ Kurzarbeit } & Insgesamt & 541,7 & $-4,0$ & 108,6 & $-6,8$ \\
\hline & Männer & 477,1 & Keine Werte & Keine Werte & Keine Werte \\
\hline & Frauen & 1207,9 & Keine Werte & Keine Werte & Keine Werte \\
\hline & West & 630,7 & $-6,9$ & 136,1 & $-8,4$ \\
\hline & Ost & 264,1 & 14,8 & 33,7 & 0,8 \\
\hline \multirow{10}{*}{$\begin{array}{l}\text { Sozialversicherungspflichtig } \\
\text { Beschäftigte }\end{array}$} & Insgesamt & 0,4 & 0,1 & $-0,6$ & $-2,0$ \\
\hline & Männer & $-0,6$ & $-0,6$ & $-1,4$ & $-2,6$ \\
\hline & Frauen & 1,7 & 1,0 & 0,5 & $-1,3$ \\
\hline & U25 & $-1,9$ & Keine Werte & $-2,0$ & $-4,8$ \\
\hline & Ü55 & 6,7 & Keine Werte & $-0,1$ & 4,5 \\
\hline & Deutsche & 0,4 & Keine Werte & $-0,5$ & $-1,9$ \\
\hline & Ausländer & 0,1 & Keine Werte & $-1,1$ & $-3,4$ \\
\hline & West & 0,4 & 0,0 & $-0,1$ & $-1,7$ \\
\hline & Ost & 0,4 & 0,7 & $-2,4$ & $-3,3$ \\
\hline & Ohne Ausbildung & $-2,8$ & Keine Werte & Keine Werte & Keine Werte \\
\hline \multirow[t]{3}{*}{ Vollzeit } & Insgesamt & $-0,4$ & $-0,6$ & $-1,3$ & $-2,7$ \\
\hline & Männer & $-0,9$ & Keine Werte & $-1,7$ & $-2,9$ \\
\hline & Frauen & 0,6 & Keine Werte & $-0,7$ & $-2,4$ \\
\hline \multirow[t]{3}{*}{ Teilzeit } & Insgesamt & 4,0 & 3,4 & 3,9 & 1,7 \\
\hline & Männer & 4,8 & Keine Werte & 5,9 & 3,8 \\
\hline & Frauen & 3,8 & Keine Werte & 3,5 & 1,4 \\
\hline \multirow{7}{*}{$\begin{array}{l}\text { Ausschließlich geringfügig } \\
\text { entlohnte Beschäftigte }\end{array}$} & Insgesamt & 0,4 & $-0,4$ & 1,5 & $-0,3$ \\
\hline & Männer & 1,7 & Keine Werte & 2,6 & 1,7 \\
\hline & Frauen & $-0,3$ & Keine Werte & 1,0 & $-1,1$ \\
\hline & Deutsche & 0,1 & Keine Werte & 1,5 & $-0,5$ \\
\hline & Ausländer & 3,7 & Keine Werte & 1,6 & 2,9 \\
\hline & West & 0,5 & $-0,6$ & 0,9 & $-1,0$ \\
\hline & Ost & $-0,8$ & 0,5 & 5,7 & 4,0 \\
\hline \multirow[t]{3}{*}{ Leiharbeiter } & Insgesamt & $-16,3$ & Keine Werte & $-12,8$ & $-0,6$ \\
\hline & Männer & $-19,3$ & Keine Werte & $-14,1$ & $-0,1$ \\
\hline & Frauen & $-8,2$ & Keine Werte & $-8,7$ & $-2,0$ \\
\hline
\end{tabular}

\section{3 \\ Auswirkungen der \\ Krise auf verschiedene \\ Personengruppen}

\subsection{FRAUEN UND MÄNNER}

\section{MÄNNER SIND STÄRKER BETROFFEN}

Die aktuelle Krise hat Männer im Hinblick auf die sozialversicherungspflichtige Beschäftigung sowie die registrierte Arbeitslosigkeit stärker getroffen als Frauen (z.B. BA 2009b). Allerdings war die Ent- wicklung bei den Männern vor der Krise besser als bei den Frauen (Abbildung 2). Das Phänomen einer stärkeren konjunkturellen Betroffenheit von Männern ist zudem nicht neu, sondern lässt sich auch für die vorangegangene Krise beobachten.

Die Entwicklung der sozialversicherungspflichtigen Beschäftigung seit $\mathrm{Be}$ ginn der Krise ist bei den Frauen eindeutig günstiger als in der vorangegangenen Krise, bei den Männern allenfalls geringfügig günstiger. Bei den Männern ging zudem in beiden Krisen die sozialversicherungspflichtige Beschäftigung zurück (Tabellen 1 und 2).
Auch bei der registrierten Arbeitslosigkeit ist die Entwicklung bei den Frauen insgesamt positiver als bei den Männern. Während bei den Männern in allen betrachteten Zeiträumen aktuell ein Anstieg $\mathrm{zu}$ beobachten ist, geht sie bei den Frauen

8 Die Quartalswerte der registrierten Arbeitslosigkeit und der Kurzarbeit wurden als Durchschnitt der drei Monate berechnet. Als Quartalswert der restlichen Daten dient jeweils der für Ende März gemessene Wert.

9 Die Quartalswerte von März 2010 der sozialversicherungspflichtigen Beschäftigten, der Voll- und Teilzeitbeschäftigten sowie der ausschließlich geringfügig entlohnten Beschäftigten liegen lediglich in hochgerechneter Form vor. 
zurück (Tabellen 1 und 2). Dies war in der vorangegangenen Krise noch nicht der Fall. Zwar war auch damals die Entwicklung der registrierten Arbeitslosigkeit bei den Frauen günstiger als bei den Männern, allerdings wiesen damals auch die Frauen eine steigende Arbeitslosigkeit auf. Sowohl für die Männer als auch für die Frauen ist die Entwicklung der registrierten Arbeitslosigkeit in der aktuellen Krise im Vergleich zur vorangegangenen günstiger.

Aufgrund der schlechteren Entwicklung bei den Männern hat deren Anteil an den registrierten Arbeitslosen in der Krise deutlich zugenommen. Während im zweiten Quartal 2008 Männer und Frauen saisonbereinigt einen etwa gleich groBen Anteil an der Arbeitslosigkeit hatten (Männer: 50,8 \%, Frauen: 49,2 \%), lag der Anteil der Männer an allen Arbeitslosen im 2. Quartal 2010 bei 54,4\%. In der letzten Krise war das gleiche Phänomen zu beobachten, allerdings waren damals generell noch mehr Männer arbeitslos als Frauen.

Auch von der krisenbedingten Kurzarbeit sind eindeutig eher Männer betroffen als Frauen. Sie hat zudem in der aktuellen Krise im Vergleich zur vorangegangenen bei den Männern stärker an Bedeutung gewonnen als bei den Frauen.

\section{BRANCHENZUGEHÖRIGKEIT ALS TEILERKLÄRUNG}

Einen wesentlichen Erklärungsgehalt für die unterschiedliche Krisenbetroffenheit von Frauen und Männern liefern deren Beschäftigtenanteile in den besonders von der Krise betroffenen Branchen. So zeigt sich, dass in den Wirtschaftszweigen, in denen relevante Beschäftigungsverluste zu verzeichnen sind, mehrheitlich Männer beschäftigt sind. Der Frauenanteil im Maschinenbau wie im Fahrzeugbau beträgt lediglich gute $15 \%$, bei der Herstellung von Gummi- und Kunststoffwaren gute $25 \%$ und bei der Herstellung von chemischen und pharmazeutischen Erzeugnissen $31 \%$ (Stand: September 2008). Die wesentliche Ausnahme stellt der Kraftfahrzeughandel mit einem Frauenanteil von über $51 \%$ dar.

In den Branchen, in denen auch nach Beginn der Krise Beschäftigungszuwächse zu verzeichnen waren, stellen hingegen die weiblichen Beschäftigten die Mehrheit dar. So liegt im Gesundheitswesen, einschließlich Heimen und Sozialwesen, der Frauenanteil bei über $80 \%$; eine Ausnahme ist der Bereich Energie- und Wasserversorgung

\begin{tabular}{|c|c|c|c|}
\hline & & 2009 zu 2008 & 2002 zu 2001 \\
\hline \multirow[t]{9}{*}{ Registrierte Arbeitslose } & Insgesamt & 4,8 & 5,4 \\
\hline & Männer & 8,5 & 12,0 \\
\hline & Frauen & $-2,8$ & 1,8 \\
\hline & U25 & 10,9 & 12,1 \\
\hline & Ü55 & 16,0 & $-15,4$ \\
\hline & Deutsche & 4,6 & 5,0 \\
\hline & Ausländer & 5,3 & 8,7 \\
\hline & West & 8,2 & 7,7 \\
\hline & Ost & $-1,8$ & 2,0 \\
\hline \multirow[t]{5}{*}{ Kurzarbeit } & Insgesamt & 1025,3 & 68,2 \\
\hline & Männer & 965,9 & Keine Werte \\
\hline & Frauen & 1316,9 & Keine Werte \\
\hline & West & 1122,2 & 72,6 \\
\hline & Ost & 610,5 & 53,9 \\
\hline \multirow{10}{*}{$\begin{array}{l}\text { Sozialversicherungspflichtige } \\
\text { Beschäftigte }\end{array}$} & Insgesamt & $-0,3$ & $-0,9$ \\
\hline & Männer & $-1,6$ & $-1,7$ \\
\hline & Frauen & 1,3 & 0,2 \\
\hline & U25 & $-3,1$ & $-3,2$ \\
\hline & Ü55 & 6,0 & 0,3 \\
\hline & Deutsche & $-0,2$ & $-0,8$ \\
\hline & Ausländer & $-1,2$ & $-1,6$ \\
\hline & West & $-0,3$ & $-0,4$ \\
\hline & Ost & 0,0 & $-2,9$ \\
\hline & ohne Berufsausbildung & $-3,7$ & Keine Werte \\
\hline \multirow[t]{3}{*}{ Vollzeit } & Insgesamt & $-1,2$ & $-1,9$ \\
\hline & Männer & $-1,8$ & $-2,2$ \\
\hline & Frauen & $-0,1$ & $-1,3$ \\
\hline \multirow[t]{3}{*}{ Teilzeit } & Insgesamt & 3,9 & 3,2 \\
\hline & Männer & 5,1 & 5,4 \\
\hline & Frauen & 3,7 & 2,8 \\
\hline \multirow{7}{*}{$\begin{array}{l}\text { Ausschließlich geringfügig } \\
\text { entlohnte Beschäftigte }\end{array}$} & Insgesamt & 0,9 & 0,6 \\
\hline & Männer & 3,1 & 1,9 \\
\hline & Frauen & $-0,1$ & 0,0 \\
\hline & Deutsche & 0,6 & 0,5 \\
\hline & Ausländer & 4,5 & 2,5 \\
\hline & West & 0,9 & 0,1 \\
\hline & Ost & 1,1 & 3,9 \\
\hline \multirow[t]{3}{*}{ Leiharbeiter } & Insgesamt & $-17,8$ & $-6,6$ \\
\hline & Männer & $-21,1$ & $-7,4$ \\
\hline & Frauen & $-8,8$ & $-3,8$ \\
\hline
\end{tabular}

mit einem Frauenanteil von lediglich $24 \%$.

Auch für die letzte Krise kann die stärkere Betroffenheit der Männer im Wesentlichen mit der Branchenzugehörigkeit erklärt werden. Beide damals besonders betroffenen Branchen - die Baubranche und die Energie- und Wasserversorgungsind eindeutig männlich dominiert.

\section{BESCHÄFTIGUNGSENTWICKLUNG FÜR MÄNNER BRANCHENINTERN SCHLECHTER}

Zwar lässt sich für die meisten Branchen mit krisenbedingten Beschäftigungsverlusten beobachten, dass die Beschäftigungsentwicklung sowohl für Frauen als auch für Männer negativ ist. Jedoch ist auffäl- lig, dass auch innerhalb dieser Branchen die Entwicklung der sozialversicherungspflichtigen Beschäftigung der Männer nach Beginn der Krise in der Regel eindeutig schlechter war als die der Frauen. Eine Ausnahme stellt die Kfz-Herstellung dar. Hier verlief die Entwicklung der sozialversicherungspflichtigen Beschäftigung bei den Frauen nach Beginn der Krise schlechter. ${ }^{10}$

In Branchen mit Beschäftigungszuwächsen sind hingegen seit Beginn der Krise kaum Unterschiede in der Beschäf-

10 Untypisch verlief die Entwicklung bei Gummi- und Kunststoffwaren. Hier nahm die sozialversicherungspflichtige Beschäftigung der Frauen nach Beginn der Krise zunächst stärker ab als die der Männer. Seit Mitte 2009 ist bei den Frauen jedoch wieder ein Anstieg zu beobachten. 
Abb. 2: Entwicklung der registrierten Arbeitslosigkeit von

Männern und Frauen

- in \%, nicht saisonbereinigt; Januar $2007=100-$

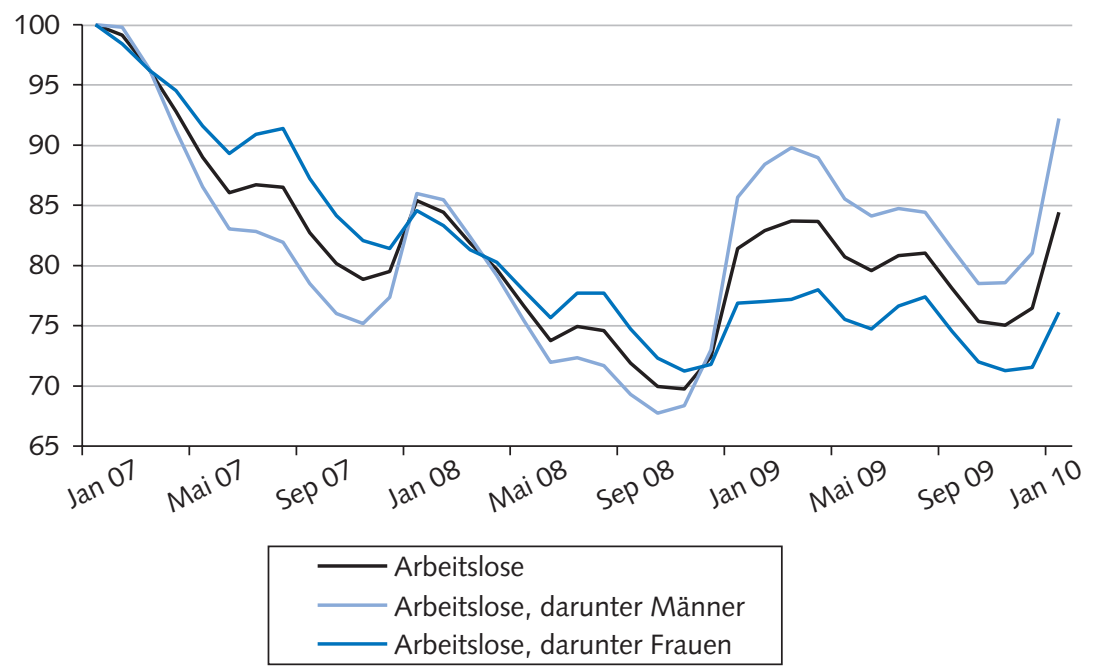

Quelle: Bundesagentur für Arbeit, Berechnungen der Autoren

WSI MITTELUNGEN

tigungsentwicklung zwischen Frauen und Männern zu beobachten. Eine Ausnahme stellt die Energie- und Wasserversorgung dar. Dort ist bei den Frauen ein deutlich stärkerer Anstieg zu verzeichnen als bei den Männern.

Die in der Regel schlechtere Beschäftigungsentwicklung von Männern innerhalb der besonders betroffenen Branchen deutet darauf hin, dass dort verschiedene Beschäftigungsbereiche in unterschiedlichem Maße von der Krise betroffen sind. So arbeiten Männer eher in der Produktion und Frauen in der Verwaltung. Eine solch differenzierte Analyse innerhalb einzelner Branchen kann jedoch im Rahmen dieses Beitrags nicht geleistet werden.

\subsection{PROBLEMGRUPPEN AM ARBEITSMARKT}

\section{BESCHÄFTIGTE OHNE BERUFS- AUSBILDUNG}

Die Beschäftigungschancen von Personen ohne Berufsausbildung haben sich in den letzten Jahren drastisch verschlechtert, während eine Ausbildung oder gar ein abgeschlossenes Studium zu einem deutlichen Absinken der Wahrscheinlichkeit führen, arbeitslos zu werden bzw. längerfristig arbeitslos zu bleiben. Gerade vor diesem Hintergrund ist es daher interessant, die Auswirkungen der Krise auf Personen ohne und mit abgeschlossener Berufsausbildung zu vergleichen. Dies kann jedoch nur für die aktuelle Krise erfolgen, da für die vorangegangene Krise keine entsprechenden Daten vorliegen.

Die sozialversicherungspflichtige Beschäftigung von Personen ohne Berufsausbildung hat im ersten Krisenjahr am stärksten abgenommen (Tabellen 1 und 2). Während die Entwicklung der sozialversicherungspflichtigen Beschäftigung bei den Personen mit Berufsausbildung ebenfalls - wenn auch in deutlich geringerem Maße - durch einen Rückgang gekennzeichnet ist, hat die sozialversicherungspflichtige Beschäftigung von Personen mit Fachhochschul- bzw. Hochschulabschluss weiter zugenommen.

\section{SOZIALVERSICHERUNGSPFLICHTIGE BESCHÄFTIGUNG VON AUSLÄNDERN}

Da in den Arbeitsmarkt- und Beschäftigungsstatistiken der BA nur nach dem Merkmal "Ausländer“ und nicht auch nach Personen mit Migrationshintergrund differenziert wird, beschränken sich die folgenden Betrachtungen auf einen Vergleich zwischen Beschäftigten und Arbeitslosen mit und ohne deutschen Pass. Das Vorliegen eines Migrationshintergrunds - unabhängig von der deutschen Staatsangehörigkeit - kann nicht berücksichtigt werden.

Die Entwicklung der sozialversicherungspflichtigen Beschäftigung verlief seit Beginn der Krise bei Personen ohne deutschen Pass schlechter als bei Personen mit deutschem Pass. Im Verlauf des ersten Krisenjahrs war bei den Personen mit deutschem Pass ein Zuwachs von 0,4 \% zu beobachten, bei den Personen ohne deutschem Pass lediglich ein Zuwachs von 0,1 \% (Tabelle 1). Noch deutlicher sind die Unterschiede im Vergleich der Jahresdurchschnitte 2008 zu 2009 (Tabelle 2). Dieses Phänomen ist keine Besonderheit der aktuellen Krise, sondern war auch schon in der vorangegangenen Krise zu beobachten.

\section{GERINGFÜGIGE BESCHÄFTIGUNG VON AUSLÄNDERN}

Im Gegensatz dazu fällt die Zunahme der ausschließlich geringfügigen Beschäftigung bei den ausländischen Personen auch nach Beginn der Krise deutlich stärker aus als bei der deutschen Bevölkerung. So lag das Jahresmittel 2009 bei den Ausländern um 4,5\% über dem Jahresmittel 2008, bei den Deutschen lediglich um 0,6\% (Tabelle 2). Während der Vergleich der beiden Krisen bei den Deutschen kaum Unterschiede in den Entwicklungen erkennen lässt (Anstieg zwischen 2001 und 2002: $0,5 \%$ ), hat bei den ausländischen Personen die geringfügige Beschäftigung in der aktuellen Krise noch etwas stärker zugenommen, als dies in der vorangegangenen Krise der Fall war (Anstieg zwischen 2001 und 2002: 2,5\%).

Der stärkere Anstieg ausschließlich geringfügiger sowie der deutlichere Rückgang an sozialversicherungspflichtiger Beschäftigung bei ausländischen Personen lassen sich u.a. dadurch erklären, dass ausländische Beschäftigte insbesondere im Bereich der Beherbergung und Gastronomie überrepräsentiert sind - einer Branche, in der trotz Krise die Beschäftigung weiter zugenommen hat, aber auch die Bedeutung der geringfügigen Beschäftigung sehr hoch ist. Es finden sich aber auch überproportional viele ausländische Beschäftigte in der Kfz-Industrie sowie in der Herstellung von Gummi- und Kunststoffwaren - beides Branchen, in denen in Folge der Krise ein Abbau von sozialversicherungspflichtiger Beschäftigung stattgefunden hat.

\section{AUSLÄNDER UND ARBEITSLOSIGKEIT}

Aktuell ist die Entwicklung der registrierten Arbeitslosigkeit bei den Ausländern im Vergleich zu den Deutschen günstiger als früher. Zwischen 2008 und 2009 ist die 
jahresdurchschnittliche Arbeitslosigkeit bei den Personen ohne deutschen Pass mit $5,3 \%$ nur geringfügig stärker angestiegen als bei den Personen mit deutscher Staatsangehörigkeit (+4,6\%). Demgegenüber lag im Vergleich der Jahresdurchschnitte 2001 und 2002 der Anstieg der registrierten Arbeitslosigkeit bei den Ausländern mit 8,7 \% deutlich über dem der deutschen Staatsangehörigen mit 5,0\% (Tabelle 2).

\section{ÄLTERE BESCHÄFTIGTE}

Mit Blick auf die über 55-Jährigen sind interessante Phänomene zu beobachten. Auch nach Beginn der Krise ist für diesen Personenkreis ein eindeutiger und überproportionaler Anstieg der sozialversicherungspflichtigen Beschäftigung festzustellen: im Verlauf des ersten Krisenjahrs um $6,7 \%$ (Tabelle 1) sowie im Vergleich der Jahresdurchschnitte 2008 und 2009 um 6,0 \% (Tabelle 2). Damit läuft die Entwicklung in der aktuellen Krise gegen den allgemeinen Trend. Zudem gilt, dass sich die sozialversicherungspflichtige Beschäftigung älterer Personen in der aktuellen Krise deutlich positiver entwickelt hat als in der vorangegangenen Krise. So war zwischen den Jahren 2001 und 2002 nur ein Anstieg der sozialversicherungspflichtigen Beschäftigung der über 55-Jährigen von $0,3 \%$ zu beobachten (Tabelle 2). Vom 1. Quartal 2001 bis zum 1. Quartal 2002 gab es sogar einen Rückgang um 0,1 \%, bis zum 1. Quartal 2003 dann wieder einen Anstieg von 4,5\% (Tabelle 1).

Gleichzeitig nimmt jedoch seit Beginn der aktuellen Krise die registrierte Arbeitslosigkeit älterer Personen stärker als die Gesamtarbeitslosigkeit zu, im Verlauf des ersten Krisenjahrs um 16,2 \% und im zweiten um weitere $10,5 \%$ (Tabelle 1) sowie im Vergleich der Jahresdurchschnitte 2008 und 2009 um 16,0 \% (Tabelle 2). Zudem hat die Verschlechterung auf dem Arbeitsmarkt vergleichsweise früh eingesetzt. Bei der registrierten Arbeitslosigkeit der Älteren ist bereits ab September 2008 - saisonbereinigt sogar ab März 2008 - eine Verschlechterung zu erkennen. Im Vergleich zur vergangenen Krise entwickelt sich die Arbeitslosigkeit bei den über 55-Jährigen aktuell deutlich ungünstiger. Damals ging sie für diese Personengruppe sogar zurück (Tabellen 1 und 2).

Diese zunächst widersprüchlichen Ergebnisse dürften zum einen auf eine deutlich gestiegene Arbeitsmarktbeteili- gung von älteren Personen im Vergleich zum Zeitraum 2001/2002 zurückzuführen sein. Zum anderen ist mittlerweile die sogenannte 58er-Regelung ausgelaufen, die ursächlich dafür war, dass viele ältere Erwerbslose noch in der vorangegangenen Krise nicht mehr in der offiziellen Arbeitslosenstatistik erfasst wurden. ${ }^{11}$

\section{JUNGE ERWERBSPERSONEN}

Ein ganz anderes Bild zeigt sich beim Personenkreis der unter 25-Jährigen: Die Entwicklung der sozialversicherungspflichtigen Beschäftigung ist hier seit Beginn der aktuellen Krise eindeutig negativ. Im Verlauf des ersten Krisenjahrs war ein Rückgang um knapp $2 \%$ zu verzeichnen (Tabelle 1), im Vergleich der Jahresdurchschnitte 2008 und 2009 sogar um mehr als $3 \%$. Allerdings entspricht diese Entwicklung ungefähr derjenigen der vorangegangen Krise.

Zudem fiel der Anstieg der Jugendarbeitslosigkeit in der aktuellen Krise weniger stark aus als in der vergangenen Krise. ${ }^{12}$ Vom 1. Quartal 2008 bis zum 1. Quartal 2009 lag der Anstieg der registrierten Arbeitslosigkeit der unter 25-Jährigen bei 2,7\% und damit weit unter dem Anstieg von 11,7 \% im Verlauf des Krisenjahrs 2001 (Tabelle 1). Auch der Anstieg zwischen den Jahren 2008 und 2009 lag mit knapp $11 \%$ unter dem Anstieg zwischen den Jahren 2001 und 2002 mit gut $12 \%$ (Tabelle 2). Im Verlauf des zweiten Krisenjahrs ist aktuell die Jugendarbeitslosigkeit bereits um 2,6 \% zurückgegangen, während sie im gleichen Zeitraum der vorangegangenen Krise noch um 10,5 \% beträchtlich anstieg (Tabelle 1).

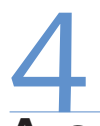

\section{Auswirkungen der Krise auf unterschiedliche Formen der Beschäftigung}

Im Mittelpunkt dieses Abschnitts steht die Frage, wie sich die aktuelle Krise auf unterschiedliche Beschäftigungsformen auswirkt. Unterscheidet sich die Entwicklung bei der Teilzeitbeschäftigung von der bei der Vollzeitbeschäftigung? Ebenso betrachten wir die Entwicklung der geringfügigen Beschäftigung mit einem Schwerpunkt auf der ausschließlich geringfügigen Beschäftigung sowie die Leiharbeit.

\subsection{TEILZEITBESCHÄFTIGUNG NIMMT ZU, VOLLZEITBESCHÄF- TIGUNG AB}

Auffällig ist, dass die sozialversicherungspflichtige Teilzeitbeschäftigung auch in der aktuellen Krise weiter zugenommen hat im Vergleich der Jahre 2008 und 2009 um rund $4 \%$. Sozialversicherungspflichtige Vollzeitbeschäftigung hat hingegen seit Beginn der Krise abgenommen - im Vergleich der Jahre 2008 und 2009 um 1,2 \% (Tabelle 2). Allerdings ist dies im Vergleich mit der vorangegangenen Krise kein neues Phänomen.

Sowohl für die Teilzeitbeschäftigung als auch für die Vollzeitbeschäftigung war die Entwicklung im Verlauf der aktuellen Krise günstiger als im Verlauf der vorangegangenen Krise. So nahm die Teilzeitbeschäftigung zwischen 2001 und 2002 nur um 3,2 \% zu. Die Vollzeitbeschäftigung nahm sogar um knapp $2 \%$ ab (Tabelle 2). Zudem war die Entwicklung der sozialversicherungspflichtigen Vollzeitbeschäftigung vor Beginn der aktuellen Krise besser als vor Beginn der letzten Krise.

Die Vollzeitbeschäftigung hat sich bei den Männern seit Beginn der Krise schlechter entwickelt als bei den Frauen. Bei der Teilzeitbeschäftigung ist für beide Geschlechter - für die Männer jedoch stärker als für die Frauen - eine Zunahme auch nach Ausbruch der Krise zu beobachten. Auch dies galt in ähnlicher Weise für die vorangegangene Krise.

\subsection{GERINGFÜGIGE BESCHÄFTIGUNG NIMMT WEITER ZU}

Die ausschließlich geringfügige Beschäftigung hatte vor Beginn der letzten Krise deutlich stärker zugenommen als vor Beginn der aktuellen Krise. Insgesamt waren bei Beginn der letzten Krise noch deutlich weniger Personen ausschließlich geringfügig beschäftigt.

Im Gegensatz zur sozialversicherungspflichtigen Beschäftigung hat die aus-

11 Unter anderem mit dieser Regelung wurden ältere Erwerbslose aus der offiziellen Arbeitslosenstatistik wegdefiniert.

12 Eine mögliche Erklärung für dieses Phänomen besteht darin, dass gleichzeitig im Jahr 2009 die Zahl der Personen in Maßnahmen zur Förderung der Berufsausbildung im Vergleich zum Vorjahr um rund 50.000 und damit um ca. $17 \%$ zugenommen hat (Quelle: Amtliche Nachrichten der Bundesagentur für Arbeit, 58. Jahrgang, Sondernummer 2). 
schließlich geringfügige Beschäftigung auch weiter zugenommen, als die aktuelle Krise sich bereits auf dem Arbeitsmarkt bemerkbar gemacht hatte. Zwischen den Jahren 2008 und 2009 war eine Zunahme von fast einem Prozent gegeben, während die sozialversicherungspflichtige Beschäftigung im gleichen Zeitraum um 0,3\% zurückgegangen ist (Tabelle 2). Die Entwicklung der ausschließlich geringfügigen Beschäftigung war in beiden Krisen recht ähnlich. Da damals jedoch die Entwicklung der sozialversicherungspflichtigen Beschäftigung schlechter war, fielen die Unterschiede in der Entwicklung beider Beschäftigungsformen in der vergangenen Krise deutlicher aus (Tabellen 1 und 2).

Die Zunahme der ausschließlich geringfügigen Beschäftigung beschränkte sich seit Herbst 2008 nur noch auf den Bereich der Dienstleistungsberufe. Im Aggregat aller anderen Berufe ist hingegen eine abnehmende Tendenz zu erkennen. Auch dies war in der vorangegangenen Krise ähnlich. Es kommt hinzu, dass die geringfügige Beschäftigung in den Dienstleistungsberufen ohnehin eine deutlich überproportionale Bedeutung hat. Somit kann das Ansteigen der geringfügigen Beschäftigung insbesondere auch darauf zurückgeführt werden, dass die Dienstleistungen von der aktuellen Krise weit weniger negativ betroffen sind als das Verarbeitende Gewerbe.

Die Zunahme der ausschließlich geringfügigen Beschäftigung in der aktuellen wie in der vorangegangenen Krise ist zudem ausschließlich auf einen Zuwachs bei den Männern zurückzuführen, während bei den Frauen jeweils eine Stagnation eintrat (Tabellen 1 und 2).

\subsection{LEIHARBEIT GEHT STARK ZURÜCK}

Im Vergleich mit der vorherigen Krise fällt der Rückgang der Leiharbeit aktuell sehr viel deutlicher aus. Im Vergleich der Jahre 2008 und 2009 war ein Rückgang von knapp $18 \%$ zu verzeichnen, im Vergleich der Jahre 2001 und 2002 lediglich um 6,6\% (Tabelle 2). Allerdings spielte die Leiharbeit auch aufgrund der damals stärkeren Regulierung im September 2001 mit 351.702 Beschäftigten noch eine weitaus geringere Rolle als im September 2008 mit 814.327 Beschäftigten, sodass die Leiharbeit inzwischen weitaus mehr sowohl für den konjunkturbedingten Beschäftigungsaufbau als auch für den konjunkturbedingten Be- schäftigungsabbau genutzt wird. Dabei fiel der Rückgang in der aktuellen Krise bei den Männern stärker aus als bei den Frauen (Tabellen 1 und 2).

\section{Schluss}

Unsere zentrale These lautete, dass nur eine Analyse der Arbeitsmarktwirkungen auf Branchen, Personengruppen und Beschäftigungsformen zeigt, was die Krise letztlich für den deutschen Arbeitsmarkt bedeutet. Dies finden wir durch die differenzierte Analyse der Entwicklung zentraler Arbeitsmarktindikatoren im Zeitablauf seit Beginn der aktuellen Krise sowie im Vergleich mit der vorangegangenen Krise bestätigt. Die gesamtwirtschaftliche Entwicklung zeigt, dass der deutsche Arbeitsmarkt insgesamt und vor allem auch im Vergleich mit der vorangegangenen Krise gut durch diese Wirtschaftskrise gekommen ist. Aber wir sehen, dass es durchaus beachtliche Unterschiede zwischen Branchen, Regionen und Personengruppen und teilweise auch beträchtliche krisenbedingte Verwerfungen - gibt.

Ausgangspunkt der aktuellen Krise war der Finanzmarktsektor - vorrangig in den USA. Zwar war auch im deutschen Finanzsektor (Kredit- und Versicherungsgewerbe) zu Beginn der Krise ein leichter Beschäftigungsabbau zu verzeichnen, es trat jedoch relativ schnell wieder eine Erholung ein. Allerdings wurde die Einschätzung gestützt, dass die Betroffenheit derjenigen Branchen besonders hoch ist, die aufgrund ihrer Investitionsintensität besonders von den Entwicklungen im Finanzmarktsektor abhängen.

Ausgehend von den gängigen Übertragungsmechanismen von Konjunkturkrisen war zudem zu vermuten, dass gerade diejenigen Branchen besonders von der Krise betroffen sind, die über Direktinvestitionen oder Exporte besonders stark von Auslandsmärkten - insbesondere dem US-amerikanischen Markt abhängig sind. Die Analysen der Arbeitsmarktwirkungen bestätigen dies: Von der aktuellen Wirtschaftskrise sind insbesondere die exportintensiven Bereiche des Verarbeitenden Gewerbes betroffen. In weiten Teilen des Dienstleistungsbereichs lassen sich hingegen auch nach Beginn der Krise weiterhin Beschäftigungszuwächse beobachten.
Zudem hat sich die eingangs formulierte These bewahrheitet, dass die regionale Betroffenheit dort besonders hoch ist, wo die export- und investitionsintensiven Branchen des Verarbeitenden Gewerbes stark vertreten sind. In der Folge sind es vor allem die starken Industriestandorte - auch im Süden und Südwesten Deutschlands, die in der Krise Beschäftigung verloren haben. Westdeutschland ist deutlich stärker von der aktuellen Krise am Arbeitsmarkt betroffen als Ostdeutschland. Dies war in der vorangegangenen Krise in diesem Ausmaß nicht der Fall.

Gemäß unseren Ausgangsüberlegungen sind Männer deutlich stärker von der Wirtschaftskrise betroffen als Frauen, da sie in stärkerem Maße als Frauen in den exportorientierten Branchen des Verarbeitenden Gewerbes beschäftigt sind. Auffällig ist allerdings, dass auch innerhalb dieser Branchen die Beschäftigungsentwicklung für die Männer schlechter verlief als für die Frauen.

Gering qualifizierte Arbeitskräfte und auch Personen mit Migrationshintergrund gehören zu den traditionellen Problemgruppen am Arbeitsmarkt. Es war daher zu vermuten, dass sie in besonderem Maße unter der Krise zu leiden haben. Tatsächlich zeigt sich, dass sich die Benachteiligung für Beschäftigte ohne abgeschlossene Berufsausbildung am Arbeitsmarkt in der Krise weiter verschärft hat. Bei Personen ohne deutschen Pass hat die sozialversicherungspflichtige Beschäftigung stärker abgenommen als bei den Deutschen, die geringfügige Beschäftigung aber hat stärker zugenommen. Im Gegensatz zur vorangegangenen Krise gab es bei der Entwicklung der registrierten Arbeitslosigkeit in der aktuellen Krise kaum Unterschiede zwischen Deutschen und Ausländern. Dies ist besonders vor dem Hintergrund bemerkenswert, dass innerhalb der ausländischen Bevölkerung die Qualifikationsstruktur ungünstiger ist als bei der deutschen Bevölkerung.

In Krisenzeiten gehen Neueinstellungen ebenso zurück wie die Ausbildungstätigkeit. Wir hatten deshalb vermutet, dass besonders die jungen (unter 25 Jahren) Arbeitsmarktteilnehmer unter der aktuellen Krise zu leiden haben. Die Entwicklung der sozialversicherungspflichtigen Beschäftigung von unter 25-Jährigen in der aktuellen wie in der vorangegangen Krise bestätigt diese Überlegung. Allerdings ist die Jugendarbeitslosigkeit aktuell nicht in dem Maße gestiegen wie damals. 
Die Ergebnisse unserer Analysen unterstützen die eingangs formulierte Hypothese, dass Ältere nicht mehr wie früher mit Hilfe von Vorruhestandsregelungen zum konjunkturbedingten Abbau der Belegschaften herangezogen werden. Ihre verstärkte Arbeitsmarktintegration hat sich auch in der Krise fortgesetzt, was sich eindrucksvoll in einer steigenden Zahl an sozialversicherungspflichtig Beschäftigten im Alter von über 55 Jahren, gleichzeitig aber auch in einer stärkeren Betroffenheit der Älteren von Arbeitslosigkeit widerspiegelt.

Der starke krisenbedingte Rückgang der Leiharbeit stützt eindeutig die Einschätzung, dass die Randbelegschaften als
Erstes und in besonderer Weise von konjunkturellen Krisen betroffen sind. Auf den ersten Blick im Widerspruch dazu steht, dass sich die ausschließlich geringfügige Beschäftigung in der Krise besser entwickelt hat als die sozialversicherungspflichtige Beschäftigung. Auf den zweiten Blick ist aber zu erkennen, dass die Zunahme der ausschließlich geringfügigen Beschäftigung vorrangig im Dienstleistungssektor stattfand, in dem die Beschäftigungsentwicklung generell positiv ausfiel und der Anteil der geringfügigen Beschäftigungsverhältnisse traditionell höher ist als im Verarbeitenden Gewerbe. Analog dazu hat auch die sozialversicherungspflichtige Teilzeitbe- schäftigung weiter zugenommen, während die Vollzeitbeschäftigung rückläufig war.

Insgesamt verstärkt die aktuelle Krise - noch in stärkerem Maße als die vorangegangene - den Strukturwandel in Richtung Dienstleistungssektor. Während sie im Hinblick auf die regionalen und geschlechtsspezifischen Unterschiede eher nivellierend wirkt, wird die Benachteiligung für Beschäftigte ohne abgeschlossene Berufsausbildung auf dem Arbeitsmarkt weiter verschärft. Gleichzeitig gehören im Vergleich zur vorangegangenen Krise Personen ohne deutschen Pass, Junge und Ältere weniger eindeutig zu den besonderen Problemgruppen.

\section{LITERATUR}

Bach, H.-U./Hummel, M./Klinger, S./Spitznagel, E./Zika, G. (2009): Arbeitsmarkt-Projektion 2010: Die Krise wird deutliche Spuren hinterlassen, IAB-Kurzbericht 20, Nürnberg

Bogedan, C./Brehmer, W./Herzog-Stein, A. (2009): Betriebliche Beschäftigungssicherung in der Krise - Eine Kurzauswertung der WSIBetriebsrätebefragung 2009, in: WSI Report 1

Bundesagentur für Arbeit (BA) (2009a): Kurzarbeit - Aktuelle Entwicklungen. Der Arbeitsmarkt in Deutschland - Arbeitsmarktberichterstattung, Nürnberg

Bundesagentur für Arbeit (BA) (2009b): Ein Jahr Krise auf dem deutschen Arbeitsmarkt. Der Arbeitsmarkt in Deutschland - Arbeitsmarktberichterstattung, Nürnberg

Crimmann, A./Wießner, F. (2009): Wirtschafts- und Finanzkrise Verschnaufpause dank Kurzarbeit, IAB-Kurzbericht 14, Nürnberg Institut der deutschen Wirtschaft (IW) (2010): Krisenbetroffenheitsindex Bayern. Welche deutschen Regionen sind am stärksten von der Krise betroffen? Bericht der IW Consult GmbH, Köln

Heckmann, M./Kettner, A./Pausch, St./Szameitat, J./Vogler-Ludwig, K. (2009): Unternehmensbefragung im II. Quartal 2009: Wie Betriebe in der Krise Beschäftigung stützen, IAB-Kurzbericht 18, Nürnberg
Möller, J.(2010): The German labor market response in the world recession - de-mystifying a miracle, in: Zeitschrift für ArbeitsmarktForschung 4, S. 325-336

Möller, J./Walwei, U. (2009): Crisis Analysis and Recommendations The German Labour Market Miracle on the Test Block, in: IAB-Forum Spezial , S. 80-87

Sachverständigenrat zur Begutachtung der gesamtwirtschaftlichen Entwicklung (SVR) (2001): Für Stetigkeit - gegen Aktionismus, Jahresgutachten 2001/2002, Wiesbaden

Sachverständigenrat zur Begutachtung der gesamtwirtschaftlichen Entwicklung (SVR) (2008): Die Finanzkrise meistern - Wachstumskräfte stärken, Jahresgutachten 2008/2009, Wiesbaden

Schwengler, B./Loibl, V. (2010): Beschäftigung, Arbeitslosigkeit und Kurzarbeit - Aufschwung und Krise wirken regional unterschiedlich, IAB-Kurzbericht 1, Nürnberg

Statistisches Bundesamt (2008): Beschäftigung und Umsatz der Betriebe des Verarbeitenden Gewerbes, Fachserie 4, Reihe 4.1.1, Wiesbaden 